\title{
Giant Brillouin Amplification in Gases
}

$\mathrm{H}$ ollow-core photonic crystal fibers have been envisioned as the backbone of future optical communications. ${ }^{1}$ These fibers promise lower transmission losses, which are associated with a lower latency, wider spectral transmission, a higher power-damage threshold, lower nonlinearities and lower dispersion than standard solid-core fibers. In 2020, the University of Southampton, U.K., reduced the loss in hollowcore fibers down to $0.28 \mathrm{~dB} / \mathrm{km}$, a value that closely approaches the loss limit in standard single-mode fibers. ${ }^{2}$

The low-loss properties of hollow-core fibers all result from a much lower interaction between photons and dense fiber material. While this dramatically reduces losses, it also makes it very difficult to affect the light via light-matter interactions-and, thus, significantly reduces the possibilities for direct optical amplification. This year, our team offered an efficient solution to this quandary.

In our work, we used stimulated Brillouin scattering directly in the gas medium (such as air) filling a hollow-core fiber to realize a massive optical amplification, by a factor of $200,000 .{ }^{3}$ We also demonstrated that the gain in a hollow-core fiber can be much stronger than in a standard single-mode fiber, and can, indeed, outperform any nonlinear gain in waveguide materials. In our experiments, we showed that the peak gain in these fibers scales with the square of the gas pressure-and turns out, at a gas pressure of only a few tens of bars, to actually exceed the Brillouin gain observed in solid materials.
Based on this principle, our team also showed that all advanced applications of stimulated Brillouin scattering-such as fiber lasing, distributed sensing and slow and fast light-can be readily implemented in hollow-core fibers, with the further advantage of a higher efficiency. We demonstrated a gas laser, based for the first time on an opto-acoustic interaction, that can operate at any wavelength, from ultraviolet to mid-infrared. Distributed sensing using hollowcore fibers showed unprecedented temperature accuracy and spatial resolution. It also offered the crucial advantage, previously unavailable in Brillouin fiber sensing, of being entirely free of strain-temperature cross-sensitivity, since the gas is insensitive to the strain applied to the fiber.

Hollow-core fibers may have crucial advantages in extreme environments, thanks to their large immunity to radiation photodarkening. Thus, the lasers and sensors demonstrated in this work may find direct application in highradiation environments, such as spacecraft or nuclear reactors. OPN

\section{RESEARCHERS}

Fan Yang, Flavien Gyger and Luc Thévenaz lluc.thevenazla epfl.ch), Ecole Polytechnique Fédérale de Lausanne, Switzerland

\section{REFERENCES}

1. R.F. Cregan et al. Science 285, 1537 (1999).

2. G.T. Jasion et al. "Hollow core NANF with $0.28 \mathrm{~dB} /$ $\mathrm{km}$ attenuation in the $\mathrm{C}$ and $\mathrm{L}$ bands," Optical Fiber Communications Conference 2020, Paper Th4B.4 (OSA, 2020).

3. F. Yang et al. Nat. Photon $14,700(2020)$.

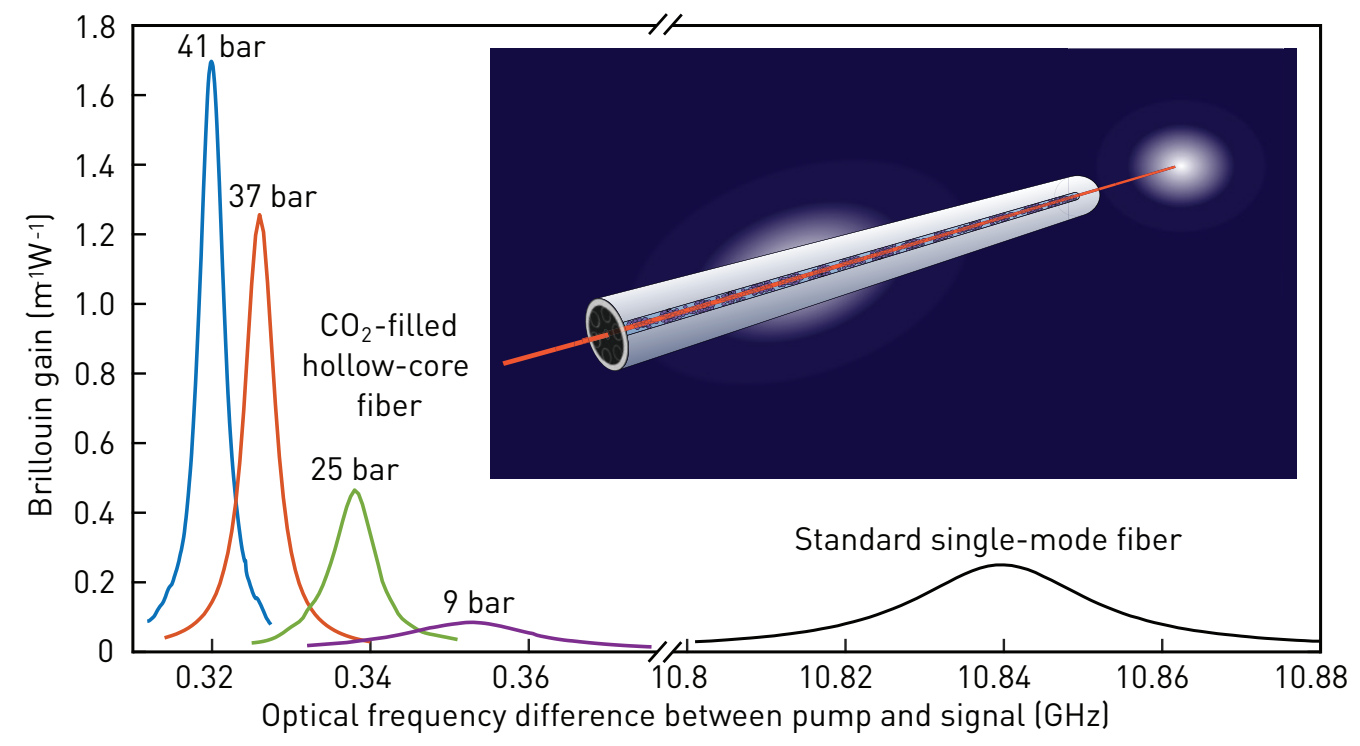

Brillouin gain obtained in a hollow-core fiber filled with $\mathrm{CO}_{2}$ gas at different pressures, compared with the gain from a solid-core silica fiber. 